\title{
Increased Expression of microRNA-17 Predicts Poor Prognosis in Human Glioma
}

\author{
Shengkui Lu, Shuai Wang, Shaomei Geng, Shucheng Ma, Zhaohui Liang, and Baohua Jiao
}

Department of Neurosurgery, Second Hospital of Hebei Medical University, No. 215 Hepingxi Road, Hebei Province, Shijiazhuang City 050000, China

Correspondence should be addressed to Baohua Jiao, jbhhbey@163.com

Received 30 September 2012; Accepted 22 October 2012

Academic Editor: Thomas Liehr

Copyright ( $(2012$ Shengkui Lu et al. This is an open access article distributed under the Creative Commons Attribution License, which permits unrestricted use, distribution, and reproduction in any medium, provided the original work is properly cited.

Aim. To investigate the clinical significance of microRNA-17 (miR-17) expression in human gliomas. Methods. Quantitative realtime polymerase chain reaction (qRT-PCR) analysis was used to characterize the expression patterns of miR-17 in 108 glioma and 20 normal brain tissues. The associations of miR-17 expression with clinicopathological factors and prognosis of glioma patients were also statistically analyzed. Results. Compared with normal brain tissues, miR-17 expression was significantly higher in glioma tissues $(P<0.001)$. In addition, the increased expression of miR-17 in glioma was significantly associated with advanced pathological grade $(P=0.006)$ and low Karnofsky performance score (KPS, $P=0.01)$. Moreover, Kaplan-Meier survival and Cox regression analyses showed that miR-17 overexpression $(P=0.008)$ and advanced pathological grade $(P=0.02)$ were independent factors predicting poor prognosis for gliomas. Furthermore, subgroup analyses showed that miR-17 expression was significantly associated with poor overall survival in glioma patients with high pathological grades (for grade III IV: $P<0.001$ ). Conclusions. Our data offer the convinced evidence that the increased expression of miR-17 may have potential value for predicting poor prognosis in glioma patients with high pathological grades, indicating that miR-17 may contribute to glioma progression and be a candidate therapeutic target for this disease.

\section{Background}

Human gliomas represent a heterogeneous group of primary malignant brain tumors, which most commonly occur in central nervous system of both children and adults [1]. These tumors are graded on the World Health Organization (WHO) grading system integrating four ascending grades of malignancy [2], as well as histologically classified as astrocytomas, oligodendrogliomas, and oligoastrocytomas [3]. Especially, the most aggressive Grade IV glioma is termed glioblastoma (GBM) and exhibits advanced features of malignancy, such as necrosis, pleomorphism, and vascular proliferation [4]. Despite ceaseless efforts by researchers to find novel therapeutic strategies, there have been no significant advances in the treatment of GBMs for several decades. The overall incidence of GBMs is about 4-5 per 100,000 persons per year, and patients with primary GBM have a clinical progression of less than about 3 months [5]. The WHO grading system reflects the anticipated malignancy of the tumor and serves as a criterion to estimate the prognosis of patients. However, clinical experiences derived from prospective randomized clinical trials indicate that histomorphological criteria alone may not be sufficient to predict clinical outcome. Gliomas with the same histopathological features may differ considerably regarding clinical course or response to therapy [6]. Undoubtedly, one reason for this is the insufficient understanding of the initiation and progression of gliomas at the molecular level. Therefore, it is of great significance to identify novel markers for gliomas in order to improve the therapeutic strategies and the individualization of therapeutic interventions.

microRNAs (miRNAs), 17-25 nucleotides endogenous noncoding single-stranded RNAs, play important roles in a wide variety of physiological and pathological processes by regulating the expression of a large number of genes posttranscriptionally [7]. Accumulating studies have indicated the involvement of miRNAs in tumorigenesis, angiogenesis, invasion, and apoptosis [8]. They are aberrantly expressed 
in many cancer types and exert tumor suppressive or oncogenic effects. To date, more than one hundred studies have demonstrated that various miRNAs are associated with tumor formation and progression of gliomas. For example, Chan et al. [9] and Papagiannakopoulos et al. [10] in 2005 both identified miR-21 as an antiapoptotic factor that targeted a network of p53, transforming growth factor (TGF)- $\beta$, and mitochondrial apoptosis tumor suppressor genes in GBM cells. Godlewski et al. [11] and Sasayama et al. [12] subsequently investigated the biological function of miR-128 and miR-10b in gliomas, respectively. In 2010, Guan et al. [13] clearly demonstrated that overexpression of miR-196a or $-196 b$ is correlated with shorter overall survival among patients with malignant glioma. In addition to be used as markers for analyzing molecular pathogenesis and classifying subtypes of human gliomas, several miRNAs have also been used as treatment targets for glioma therapy. For example, Verhaak et al. [14] in 2010 revealed that miR-128 may be used to repress the growth of glioma initiating cell in vivo, indicating its therapeutic effect of suppressing proliferation and enhancing differentiation of glioma initiating cells. Taken together, the extensive involvement of miRNAs in the regulation of various cancerrelated genes' expressions may be the molecular mechanisms of glioma initiation and progression and can be used as treatment agents or targets for the therapy of this disease.

The miR-17-92 cluster is one of the polycistronic miRNA clusters and includes six members: miR-17, miR-18a, miR19a/b, miR-20a, and miR-92a [15]. This cluster is contained within c13orf25 variant 2 , indicating that c13orf25 is a primiRNA of the mature miRNA-17-92 [16]. Recent studies have demonstrated the important roles of the miR-1792 cluster in tumorigenesis. Upregulation of entire miR17-92 cluster in myc-induced cancers may increase tumor angiogenesis by a paracrine mechanism [17]. This proangiogenic function has been attributed to the downregulation of the antiangiogenic molecules, which are targeted by miR-18 and miR-19 [18]. The present study is focused on miR-17, an important component of the miR-17-92 cluster. Accumulating studies have indicated that miR-17 functions as an oncogene or tumor suppressor in different cancer types. For example, the overexpression of the miR-17 polycistron has been detected in lung cancers [19]; miR-17 could control cellular proliferation and apoptosis of tumor cells by targeting the E2F family of transcription factors and by downregulating the tumor suppressor p21 and the proapoptotic protein BIM [20]; however, the overexpressed miR-17 may suppress the proliferation of breast cancer cells by repressing the expression of AIB1 (amplified in breast cancer-1) and CCND1 [21]. To our interests, previous studies also reported that miR-17 was upregulated in GBM tissues and might be involved in the viability, proliferation, apoptosis, and invasive growth properties of glioma cells in vitro [22]. However, the clinical significance of this miRNA in patients with gliomas has not been fully elucidated. In this study, we would like to address this problem.

\section{Materials and Methods}

2.1. Patients and Tissue Samples. This study was approved by the Research Ethics Committee of Second Hospital of Hebei Medical University, P. R. China. Written informed consent was obtained from all of the patients. All specimens were handled and made anonymous according to the ethical and legal standards.

One hundred and eight human glioma tissue samples for qRT-PCR were obtained from Department of Neurosurgery, Second Hospital of Hebei Medical University. Samples were quickly removed at surgery and immediately divided into two parts; one part was fixed in $4 \%$ paraformaldehyde for 24 hours, paraffin embedded, and used for histopathological diagnosis, and the remaining part was snap frozen in liquid nitrogen and maintained at $-80^{\circ} \mathrm{C}$ until used for RNA isolation. All the slides were reevaluated according to WHO classifications [2] by two pathologists, with differences resolved by careful discussion. A total of 64 males and 44 females $(1.45: 1)$ were enrolled in this study, and the median age was 43 years (range, 13-72). Thirty of the 108 gliomas were classified as low-grade (18 pilocytic astrocytomas (WHO I) and 12 diffuse astrocytomas (WHO II)), and 78 were classified as high-grade gliomas (32 anaplasia astrocytomas (WHO III), and 46 primary glioblastomas (WHO IV)). None of the patients had received chemotherapy or radiotherapy prior to surgery. The clinicopathological features and the treatment strategies of all patients were indicated in Table 1. Twenty normal brain tissue samples used as controls were obtained by collecting donations with consents from individuals who died in traffic accidents and were confirmed to be free of any prior pathological lesions.

All patients had complete five-year followup until death. Overall survival time was calculated from the date of the initial surgical operation to death. Patients, who died of diseases not directly related to their gliomas or due to unexpected events, were excluded from this study.

\subsection{RNA Extraction and $q R T-P C R$ for miRNA Detection.} Total RNA from fresh glioma and normal brain tissues was isolated with TRIzol reagent according to the manufacture's instruction. Synthesis of cDNA with reverse transcriptase was performed by NCode miRNA quantitative RT-PCR Kits (Invitrogen). qRT-PCR was performed using TaqMan microRNA Assay primer with the TaqMan Universal PCR Master Mix and analyzed with an ABI Prism 7000 Sequence Detection System (Applied Biosystems; Foster City, CA, USA) according to the manufacturer's instructions. Analysis was performed by the comparative threshold cycle $(\mathrm{Ct})$ method according to User Bulletin no. 2 (Applied Biosystems). Each sample was examined in triplicate and the amounts of the PCR products produced were normalized to U6B which served as internal control.

2.3. Statistical Analysis. All computations were carried out using the software of SPSS version 13.0 for Windows (SPSS Inc, IL, USA). Data were expressed as means \pm standard deviation (SD). The differential expression of miR-17 between 


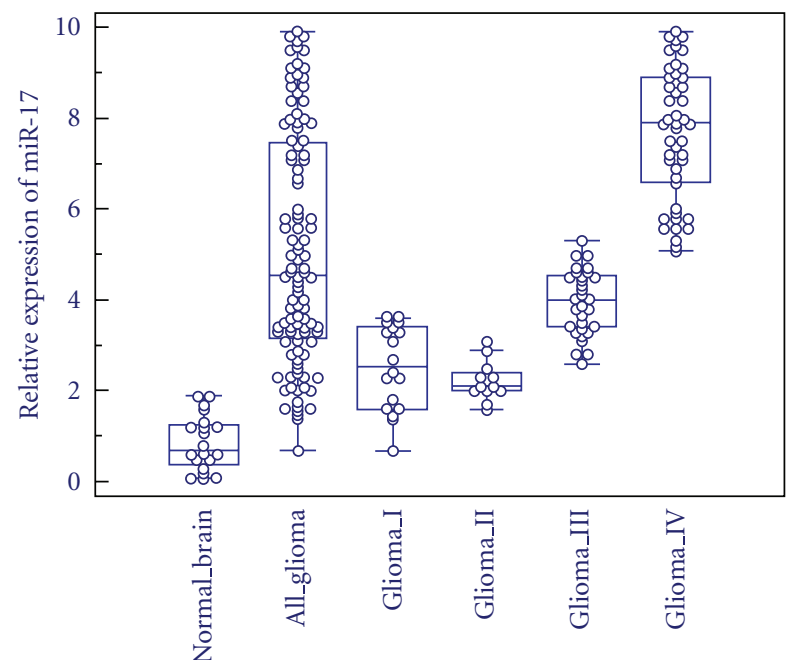

FIGURE 1: microRNA-17 (miR-17) expression in 108 glioma and 20 normal brain tissues detected by quantitative real-time polymerase chain reaction (qRT-PCR) analysis. Compared with normal brain tissues, miR-17 expression was significantly higher in glioma tissues (mean \pm SD: $5.1 \pm 2.5$ versus $0.9 \pm 0.6, P<0.001$, Figure 1 ), corresponding to the glioma WHO grades. The statistic results showed that its expression in high-grade (III-IV; mean \pm SD: $6.2 \pm$ 2.2 ) and low-grade (I-II; mean \pm SD: $2.4 \pm 0.8$ ) gliomas were both significantly higher than that in normal brains tissues (both $P<$ $0.001)$. Moreover, there was also a significant difference in miR-17 expression between high-grade (III-IV) and low-grade (I-II) glioma tissue specimens $(P<0.001)$.

glioma tissues and normal brain tissues was evaluated by independent sample $t$-test. The $\chi^{2}$ test was used to analyze the relationship between miR-17 expression and the clinicopathological characteristics. A life table was calculated according to the Kaplan-Meier method. Hazard ratios for the time-to-event endpoint were estimated using the multivariate Cox regression analysis in a forward stepwise method to evaluate the effect of multiple independent prognostic factors on survival outcome. Differences were considered statistically significant when $P$ was less than 0.05 .

\section{Results}

3.1. miR-17 Upregulation in Human Glioma Tissues. The expression levels of miR-17 were detected in 108 glioma and 20 normal brain tissues normalized to U6B. Compared with normal brain tissues, miR-17 expression was significantly higher in glioma tissues (mean \pm SD: $5.1 \pm 2.5$ versus $0.9 \pm 0.6$, $P<0.001$, Figure 1), corresponding to the glioma WHO grades. The statistic results showed that its expressions in high-grade (III-IV; mean \pm SD: $6.2 \pm 2.2$ ) and low-grade (I$\mathrm{II}$; mean $\pm \mathrm{SD}: 2.4 \pm 0.8)$ gliomas were both significantly higher than that in normal brains tissues (both $P<0.001$ ). Additionally, there was also a significant difference in miR-17 expression between high-grade (III-IV) and low-grade (I-II) glioma tissue specimens $(P<0.001)$.

3.2. miR-17 Upregulation Associates with Aggressive Clinicopathological Features of Human Gliomas. According to the relative expression levels of miR-17 to those of U6B, all 108 glioma tissue samples were divided into two groups: high miR-17 expression group (expressing miR-17 at levels more than the median expression level (5.1), mean expression value 6.9, $n=60$ ) and low miR-17 expression group (expressing miR-17 at levels less than the median expression level (5.1), mean expression value 2.9, $n=48$ ). Then, the association of miR-17 expression with various clinicopathological parameters of glioma tissues was analyzed as shown in Table 1 . The increased expression of miR-17 was significantly more common in glioma tissues with advanced pathological grade than those with low pathological grade $(P=0.006)$. A significant relationship was also found between miR17 expression and Karnofsky performance score (KPS). The overexpression of miR-17 more frequently occurred in tumors with low KPS than those with high $\operatorname{KPS}(P=$ 0.01 , Table 1). However, there was no significant association between miR-17 expression and other clinicopathological parameters, including patients' gender and age at diagnosis, and tumor size (all $P>0.05$, Table 1$)$.

3.3. miR-17 Upregulation Predicts Poor Overall Survival in Patients with Gliomas. Furthermore, the association of miR17 expression with prognosis in patients with gliomas was determined. The detail clinical information of all 108 glioma patients in high miR-17 expression and low miR-17 expression groups was reviewed. According to the log-rank test and Kaplan-Meier analysis, the expression level of miR17 in gliomas significantly displayed a correlation with the patients' survival time. Interestingly, the overall survival of patients whose tumors expressed high levels of miR-17 was significantly shorter than those with low levels of miR-17 $(P=0.001$, Figure $2(\mathrm{a}))$.

Univariate and multivariate analyses were performed and determined whether the miR-17 expression level and various clinical parameters were independent prognostic factors of patient outcomes. As the results in Table 2 show, miR17 overexpression $(P=0.008)$ and advanced pathological grade $(P=0.02)$ were independent factors predicting poor prognosis for gliomas.

We further analyzed the prognostic value of miR-17 expression in selective patient subgroups stratified according to the WHO classification. MiR-17 expression was significantly associated with poor overall survival in glioma patients with high pathological grades (for grade III $\sim$ IV: $P<0.001$; Table 3, Figures 2(b) and 2(c)) but was not as significant as in patients with low pathological grades (for grade I II: $P=0.1$; Table 3 , Figures $2(\mathrm{~b})$ and $2(\mathrm{c})$ ).

\section{Discussion}

Human gliomas are the most lethal neurological cancers. Despite research efforts, the prognosis for patients with malignant gliomas remains poor. Thus, the advances in the understanding of cellular and molecular alterations in gliomas and the development of novel, molecularly targeted therapeutic agents for its treatment are urgently needed. It has been demonstrated that miRNAs regulate the expression 


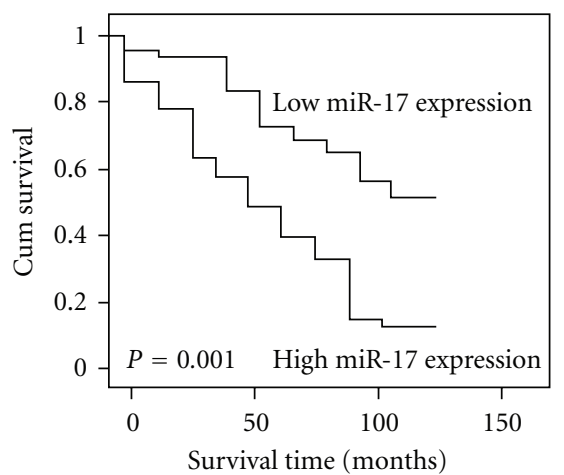

(a)

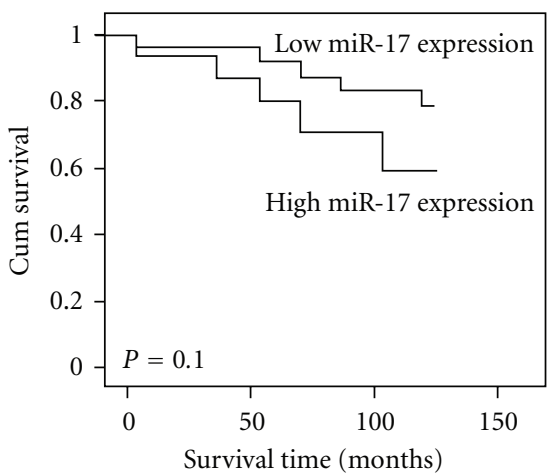

(b)

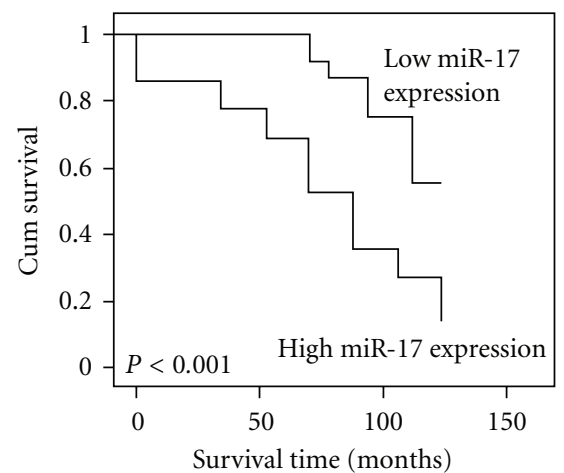

(c)

FIGURE 2: Kaplan-Meier survival curves for glioma patients in high and low microRNA-17 (miR-17) expression groups. (a) The 5-year overall survival rate of glioma patients with high miR-17 expression was significantly lower than those with low miR-17 expression $(P=0.001)$. (b) The 5-year overall survival rate of grade I II glioma patients with high miR-17 expression had no significant differences from those with low miR-17 expression $(P=0.1)$. (c) The 5-year overall survival rate of grade III $\sim$ IV glioma patients with high miR-17 expression was significantly lower than those with low miR-17 expression $(P<0.001)$.

TABLE 1: Association of miR-17 expression in human glioma tissues with different clinicopathological features.

\begin{tabular}{|c|c|c|c|c|}
\hline \multirow{2}{*}{ Clinicopathological features } & \multirow{2}{*}{ No. of cases } & \multicolumn{2}{|c|}{ miR-17 expression } & \multirow{2}{*}{$P$} \\
\hline & & $\operatorname{High}(n, \%)$ & Low $(n, \%)$ & \\
\hline \multicolumn{5}{|l|}{ WHO grade } \\
\hline I & 18 & $0(0)$ & $18(100.0)$ & \multirow{4}{*}{0.006} \\
\hline II & 12 & $1(8.3)$ & $11(91.7)$ & \\
\hline III & 32 & $20(62.5)$ & $12(37.5)$ & \\
\hline IV & 46 & $39(84.8)$ & $7(15.2)$ & \\
\hline \multicolumn{5}{|l|}{ Age } \\
\hline$<50$ & 42 & $18(42.9)$ & $24(57.1)$ & \multirow{2}{*}{ NS } \\
\hline$\geq 50$ & 66 & $42(63.6)$ & $24(36.4)$ & \\
\hline \multicolumn{5}{|l|}{ Gender } \\
\hline Male & 64 & $39(60.9)$ & $25(39.1)$ & \multirow{2}{*}{ NS } \\
\hline Female & 44 & $21(47.7)$ & $23(52.3)$ & \\
\hline \multicolumn{5}{|l|}{ Tumor size } \\
\hline$\geq 6 \mathrm{~cm}$ & 75 & $40(53.3)$ & $35(46.7)$ & \multirow{2}{*}{ NS } \\
\hline$<6 \mathrm{~cm}$ & 33 & $20(60.6)$ & $13(39.4)$ & \\
\hline \multicolumn{5}{|l|}{ KPS } \\
\hline$<90$ & 78 & $54(69.2)$ & $24(30.8)$ & \multirow{2}{*}{0.01} \\
\hline$\geq 90$ & 30 & $6(20.0)$ & $24(80.0)$ & \\
\hline
\end{tabular}

TABLE 2: Univariate and multivariate analyses of different prognostic parameters in patients with gliomas by Cox regression analysis.

\begin{tabular}{|c|c|c|c|c|c|c|}
\hline \multirow{2}{*}{ Parameter } & \multicolumn{3}{|c|}{ Univariate analysis } & \multicolumn{3}{|c|}{ Multivariate analysis } \\
\hline & Risk ratio & $95 \%$ confidence interval & $P$ & Risk ratio & 95\% confidence interval & $P$ \\
\hline Age & 1.8 & $0.5-3.8$ & 0.6 & 1.2 & $0.2-3.3$ & 0.8 \\
\hline Gender & 2.0 & $0.7-4.3$ & 0.2 & 1.6 & $0.3-3.9$ & 0.3 \\
\hline WHO grade & 4.2 & $1.3-12.0$ & 0.006 & 3.7 & $0.9-10.1$ & 0.02 \\
\hline Tumor size & 2.3 & $0.9-4.7$ & 0.1 & 1.3 & $0.9-3.7$ & 0.2 \\
\hline KPS & 2.4 & $1.0-4.8$ & 0.1 & 1.3 & $1.0-3.8$ & 0.2 \\
\hline miR-17 expression & 6.2 & $1.3-18.6$ & 0.001 & 5.1 & $0.8-15.9$ & 0.008 \\
\hline
\end{tabular}


TABLE 3: Subgroup log-rank analysis of miR-17 expression and prognosis in patients with different pathological grades.

\begin{tabular}{lccc}
\hline WHO grade & miR-17 expression status & 5-year overall survival (\%) & $P$ \\
\hline \multirow{2}{*}{ I $\sim$ II } & High & 60.0 & 0.1 \\
& Low & 80.0 & $<0.001$ \\
\hline \multirow{2}{*}{ III $\sim$ IV } & High & 18.2 & 52.2 \\
\end{tabular}

of one-third of the human genome [23]. Therefore, it is no doubt that they are involved in many aspects of glioma tumorigenesis and advancement. In this context, our research group intended to identify novel miRNA markers for the diagnosis and prognosis in human gliomas. In this study, we focus on miR-17. There are four points of our findings. Firstly, miR-17 was upregulated in human glioma tissues compared with normal brain tissues. Secondly, the increased miR-17 expression in glioma tissues was significantly correlated with advanced tumor progression and aggressive clinicopathological features. Thirdly, the results of Kaplan-Meier analyses showed that glioma tissues with high miR-17 expression tend to have unfavorable overall survival. Finally, the multivariate analysis clearly demonstrated that high miR-17 expression was a statistically significant risk factor affecting overall survival in glioma patients, especially for high grade tumors, suggesting that miR-17 expression could be a valuable marker of glioma progression and prognosis. To our knowledge, this is the first study to analyze the clinical significance of miR-17 in a large number of glioma patients.

The miR-17-92 cluster is highly conserved throughout species and has evolved along with vertebrates [24]. Through evolution, a series of duplications, deletions, and mutations have given rise to the modern miR-17-92 cluster, which is located on human chromosome 13 [25]. This cluster plays important roles in embryonic development and embryonic stem cell proliferation and differentiation [26]. Gene duplications and deletions eventually resulted in two miR-17-92 paralogues, the miR-106b-25 cluster on chromosome 7 and the miR-106a-363 cluster on chromosome X [27]. Because of their ability to suppress expression of various cancerrelated genes, the miR-17-92 cluster and its paralogues have been demonstrated to exert crucial functions in cancers. Transcription of this cluster is transactivated by the oncogene c-Myc [17]. The overexpression of entire miR-17-92 cluster has been observed in hematopoietic malignancies, breast, colon, and lung cancers, medulloblastoma, glioblastoma, and neuroblastoma [28-30]. The oncogenic nature of miR-1792 activation is supported by the identification of miR-1792 targets with key roles in cell-cycle control and cell death. Among members of the miR-17-92 cluster, miR-17 is the most extensively studied one because both oncogenic and tumor suppressive functions have been attributed to this miRNA. In line with its oncogenic role, inhibition of miR-17 upregulation in colon cancer cells could reduce cell proliferation, induce arrest of cells in the G1/S stage, and suppress tumor growth by regulating RND3, a negative regulator of cell proliferation and cell cycle progression [31]. miR-17 upregulation in B-cell lymphomas could promote tumor growth by targeting E2F1 and may increase angiogenesis by targeting thrombospondin-1 [32]. The levels of miR-17 in peripheral blood mononuclear cells from patients with gastric cancer were significantly higher than those from healthy volunteers, and the increased level of this miRNA suggested that there are tumor cells in the peripheral blood from patients with gastric cancer [33]. In the present study, we observed the overexpression of miR-17 in glioma tissues with ascending order of WHO grades, which was consistent with the findings of Malzkorn et al. [22]. In addition to the aberrant expression patterns of miR-17, we also found that the upregulation of this miRNA was associated with advanced tumor progression and poor overall survival of gliomas. Malzkorn et al. [22] have demonstrated that the inhibition of miR-17 significantly reduced cell viability and increased apoptotic activity in glioma cell lines, which may be the reasons for the relationship between upregulation of miR-17 and aggressive clinicopathological features of glioma patients.

In conclusion, our data offer the convinced evidence that the increased expression of miR-17 may have potential value for predicting poor prognosis in glioma patients with high pathological grades, indicating that miR-17 may contribute to glioma progression and be a candidate therapeutic target for this disease. However, the mechanism by which miR17 was upregulated in gliomas is still unclear. This study is hypothesis generating, and that further prospective analysis should be worth doing.

\section{Conflict of Interests}

The authors declare that they have no conflict of interests.

\section{References}

[1] T. Marumoto and H. Saya, "Molecular biology of glioma," Advances in Experimental Medicine and Biology, vol. 746, pp. 2-11, 2012.

[2] A. Rousseau, K. Mokhtari, and C. Duyckaerts, "The 2007 WHO classification of tumors of the central nervous systemWhat has changed?" Current Opinion in Neurology, vol. 21, no. 6, pp. 720-727, 2008.

[3] A. A. Brandes, "State-of-the-art treatment of high-grade brain tumors," Seminars in Oncology, vol. 30, no. 6, pp. 4-9, 2003.

[4] H. Ohgaki and P. Kleihues, "Epidemiology and etiology of gliomas," Acta Neuropathologica, vol. 109, no. 1, pp. 93-108, 2005.

[5] M. Hutterer, P. Knyazev, A. Abate et al., "Axl and growth arrestspecific gene 6 are frequently overexpressed in human gliomas and predict poor prognosis in patients with glioblastoma 
multiforme," Clinical Cancer Research, vol. 14, no. 1, pp. 130$138,2008$.

[6] G. Tabatabai, M. Hegi, R. Stupp, and M. Weller, "Clinical implications of molecular neuropathology and biomarkers for malignant glioma," Current Neurology and Neuroscience Reports, vol. 12, no. 3, pp. 302-307, 2012.

[7] R. Visone and C. M. Croce, "MiRNAs and cancer," American Journal of Pathology, vol. 174, no. 4, pp. 1131-1138, 2009.

[8] M. Katakowski, B. Buller, X. Wang, T. Rogers, and M. Chopp, "Functional microRNA is transferred between glioma cells," Cancer Research, vol. 70, no. 21, pp. 8259-8263, 2010.

[9] X. Chan, S. Nama, F. Gopal et al., "Targeting glioma stem cells by functional inhibition of a prosurvival oncomiR-138 in malignant gliomas," Cell Reports, vol. 2, no. 3, pp. 591-602, 2012.

[10] T. Papagiannakopoulos, A. Shapiro, and K. S. Kosik, "MicroRNA-21 targets a network of key tumor-suppressive pathways in glioblastoma cells," Cancer Research, vol. 68, no. 19, pp. 8164-8172, 2008.

[11] J. Godlewski, M. O. Nowicki, A. Bronisz et al., "Targeting of the Bmi-1 oncogene/stem cell renewal factor by microRNA128 inhibits glioma proliferation and self-renewal," Cancer Research, vol. 68, no. 22, pp. 9125-9130, 2008.

[12] T. Sasayama, M. Nishihara, T. Kondoh, K. Hosoda, and E. Kohmura, "MicroRNA-10b is overexpressed in malignant glioma and associated with tumor invasive factors, uPAR and RhoC," International Journal of Cancer, vol. 125, no. 6, pp. 1407-1413, 2009.

[13] Y. Guan, M. Mizoguchi, K. Yoshimoto et al., "MiRNA-196 is upregulated in glioblastoma but not in anaplastic astrocytoma and has prognostic significance," Clinical Cancer Research, vol. 16, no. 16, pp. 4289-4297, 2010.

[14] R. G. W. Verhaak, K. A. Hoadley, E. Purdom et al., "Integrated genomic analysis identifies clinically relevant subtypes of glioblastoma characterized by abnormalities in PDGFRA, IDH1, EGFR, and NF1," Cancer Cell, vol. 17, no. 1, pp. 98$110,2010$.

[15] K. Sasaki, G. Kohanbash, A. Hoji et al., "MiR-17-92 expression in differentiated T cells-implications for cancer immunotherapy," Journal of Translational Medicine, vol. 8, article 17, 2010.

[16] T. Uziel, F. V. Karginov, S. Xie et al., "The miR-17 92 cluster collaborates with the Sonic Hedgehog pathway in medulloblastoma," Proceedings of the National Academy of Sciences of the United States of America, vol. 106, no. 8, pp. 2812-2817, 2009.

[17] H. Tagawa, K. Karube, S. Tsuzuki, K. Ohshima, and M. Seto, "Synergistic action of the microRNA-17 polycistron and Myc in aggressive cancer development," Cancer Science, vol. 98, no. 9, pp. 1482-1490, 2007.

[18] K. Tréguer, E.-M. Heinrich, K. Ohtani, A. Bonauer, and S. Dimmeler, "Role of the microRNA-17-92 cluster in the endothelial differentiation of stem cells," Journal of Vascular Research, vol. 49, no. 5, pp. 447-460, 2012.

[19] H. Osada and T. Takahashi, "let-7 and miR-17-92: small-sized major players in lung cancer development," Cancer Science, vol. 102, no. 1, pp. 9-17, 2011.

[20] L. Fontana, M. E. Fiori, S. Albini et al., "Antagomir-17$5 \mathrm{p}$ abolishes the growth of therapy-resistant neuroblastoma through p21 and BIM," PLoS One, vol. 3, no. 5, Article ID e2236, 2008.

[21] S. Volinia, G. A. Calin, C. G. Liu et al., "A microRNA expression signature of human solid tumors defines cancer gene targets," Proceedings of the National Academy of Sciences of the United States of America, vol. 103, no. 7, pp. 2257-2261, 2006.

[22] B. Malzkorn, M. Wolter, F. Liesenberg et al., "Identification and functional characterization of microRNAs involved in the malignant progression of gliomas," Brain Pathology, vol. 20, no. 3, pp. 539-550, 2010.

[23] Y. Zhang, A. Dutta, and R. Abounader, "The role of microRNAs in glioma initiation and progression," Frontiers in Bioscience, vol. 17, no. 2, pp. 700-712, 2012.

[24] A. Tanzer and P. F. Stadler, "Molecular evolution of a microRNA cluster," Journal of Molecular Biology, vol. 339, no. 2, pp. 327-335, 2004.

[25] A. Ventura, A. G. Young, M. M. Winslow et al., "Targeted deletion reveals essential and overlapping functions of the miR-17 92 family of miRNA clusters," Cell, vol. 132, no. 5, pp. 875-886, 2008.

[26] K. M. Foshay and G. I. Gallicano, "miR-17 family miRNAs are expressed during early mammalian development and regulate stem cell differentiation," Developmental Biology, vol. 326, no. 2, pp. 431-443, 2009.

[27] S. W. Shan, D. Y. Lee, Z. Deng et al., "MicroRNA MiR-17 retards tissue growth and represses fibronectin expression," Nature Cell Biology, vol. 11, no. 8, pp. 1031-1038, 2009.

[28] W. Liu, Y. H. Gong, T. F. Chao et al., "Identification of differentially expressed microRNAs by microarray: a possible role for microRNAs gene in medulloblastomas," Chinese Medical Journal, vol. 122, no. 20, pp. 2405-2411, 2009.

[29] A. Ernst, B. Campos, J. Meier et al., "De-repression of CTGF via the miR-17-92 cluster upon differentiation of human glioblastoma spheroid cultures," Oncogene, vol. 29, no. 23, pp. 3411-3422, 2010.

[30] P. A. Northcott, A. Fernandez-L, J. P. Hagan et al., "The miR$17 / 92$ polycistron is up-regulated in sonic hedgehog-driven medulloblastomas and induced by N-myc in sonic hedgehogtreated cerebellar neural precursors," Cancer Research, vol. 69, no. 8, pp. 3249-3255, 2009.

[31] H. Luo, J. Zou, Z. Dong, Q. Zeng, D. Wu, and L. Liu, "Upregulated miR-17 promotes cell proliferation, tumour growth and cell cycle progression by targeting the RND3 tumour suppressor gene in colorectal carcinoma," Biochemical Journal, vol. 442, no. 2, pp. 311-321, 2012.

[32] J. K. Molitoris, K. S. McColl, and C. W. Distelhorst, "Glucocorticoid-mediated repression of the oncogenic microRNA cluster miR-17-92 contributes to the induction of bim and initiation of apoptosis," Molecular Endocrinology, vol. 25, no. 3, pp. 409-420, 2011.

[33] H. Zhou, J. M. Guo, Y. R. Lou et al., "Detection of circulating tumor cells in peripheral blood from patients with gastric cancer using microRNA as a marker," Journal of Molecular Medicine, vol. 88, no. 7, pp. 709-717, 2010. 

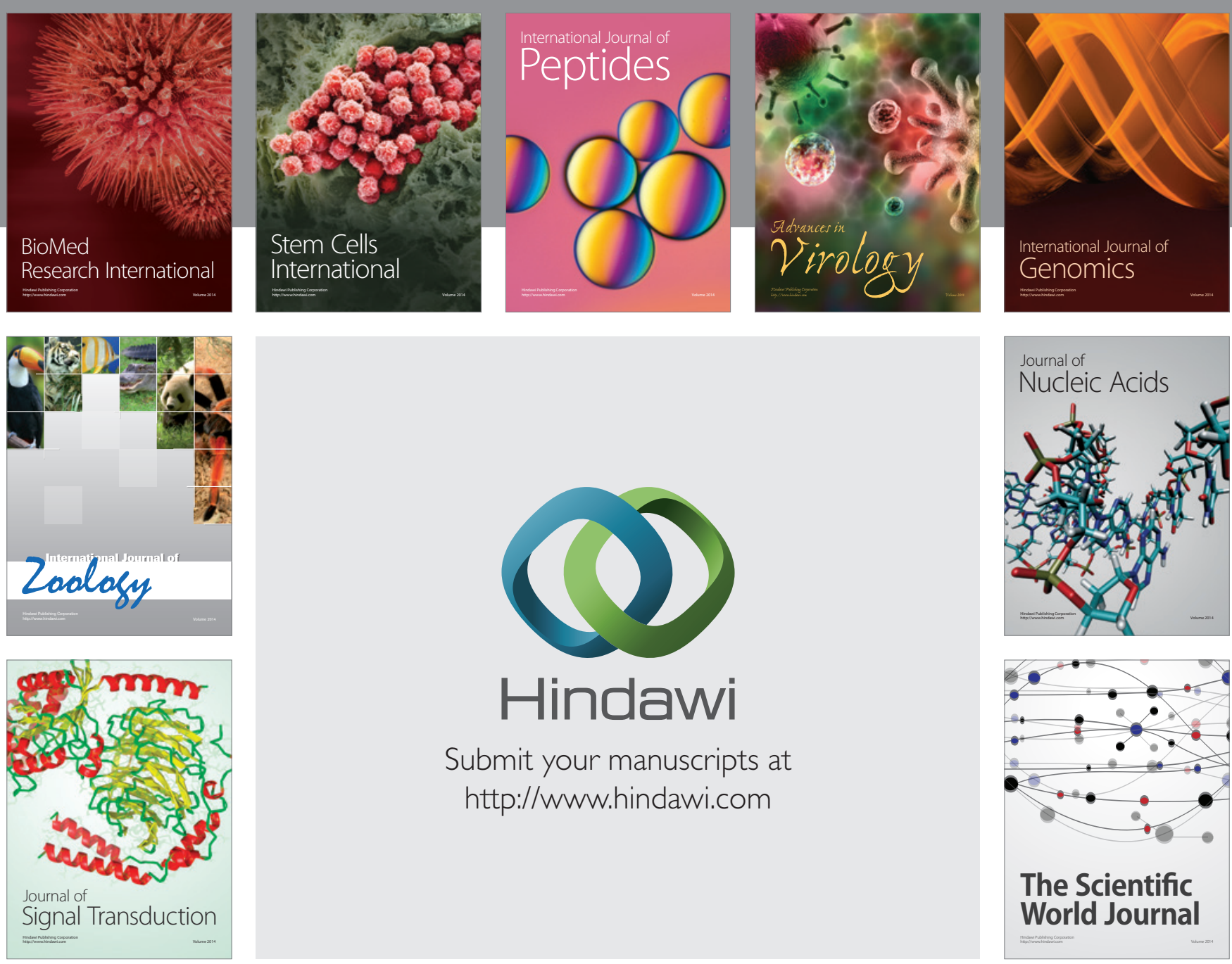

Submit your manuscripts at

http://www.hindawi.com
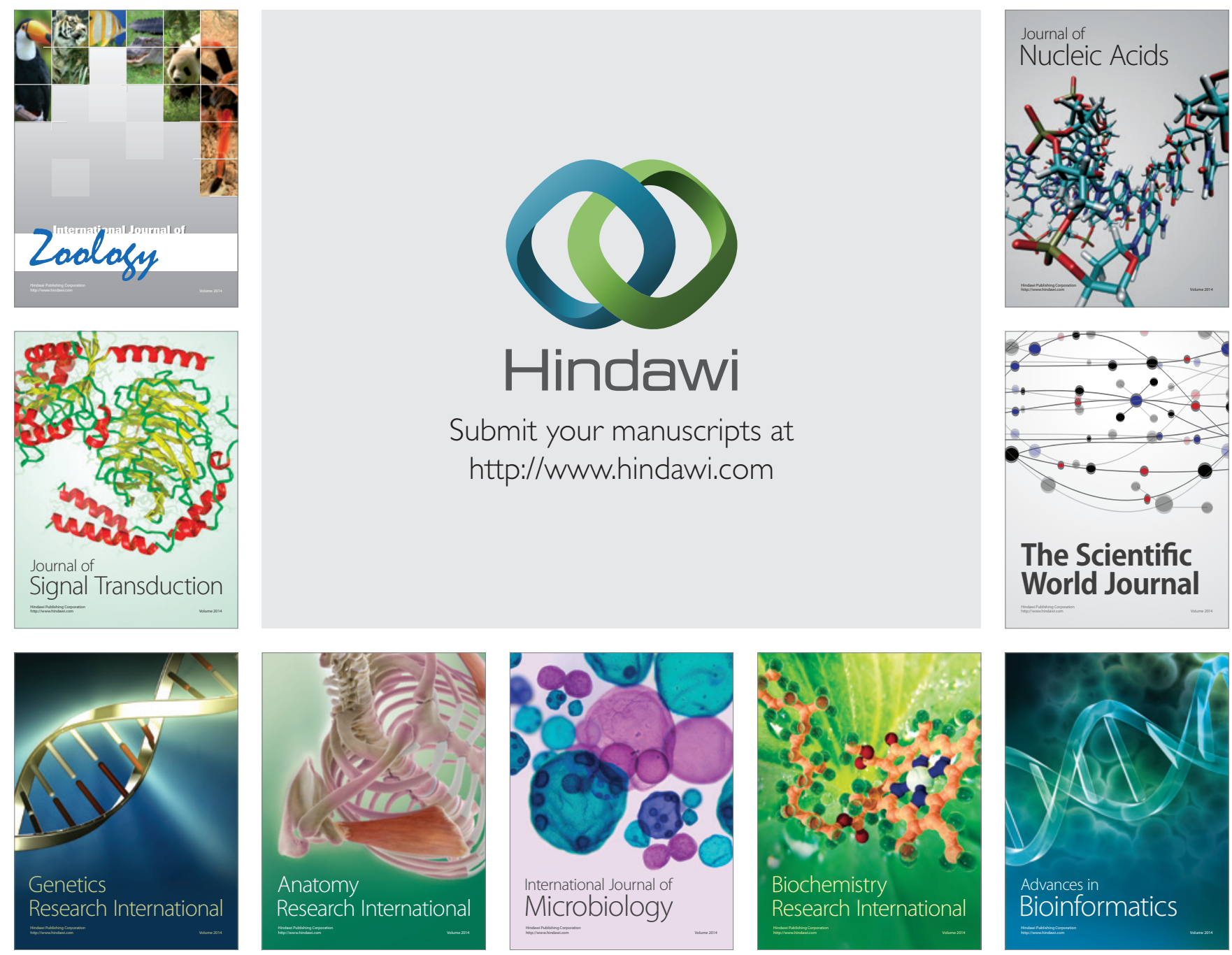

The Scientific World Journal
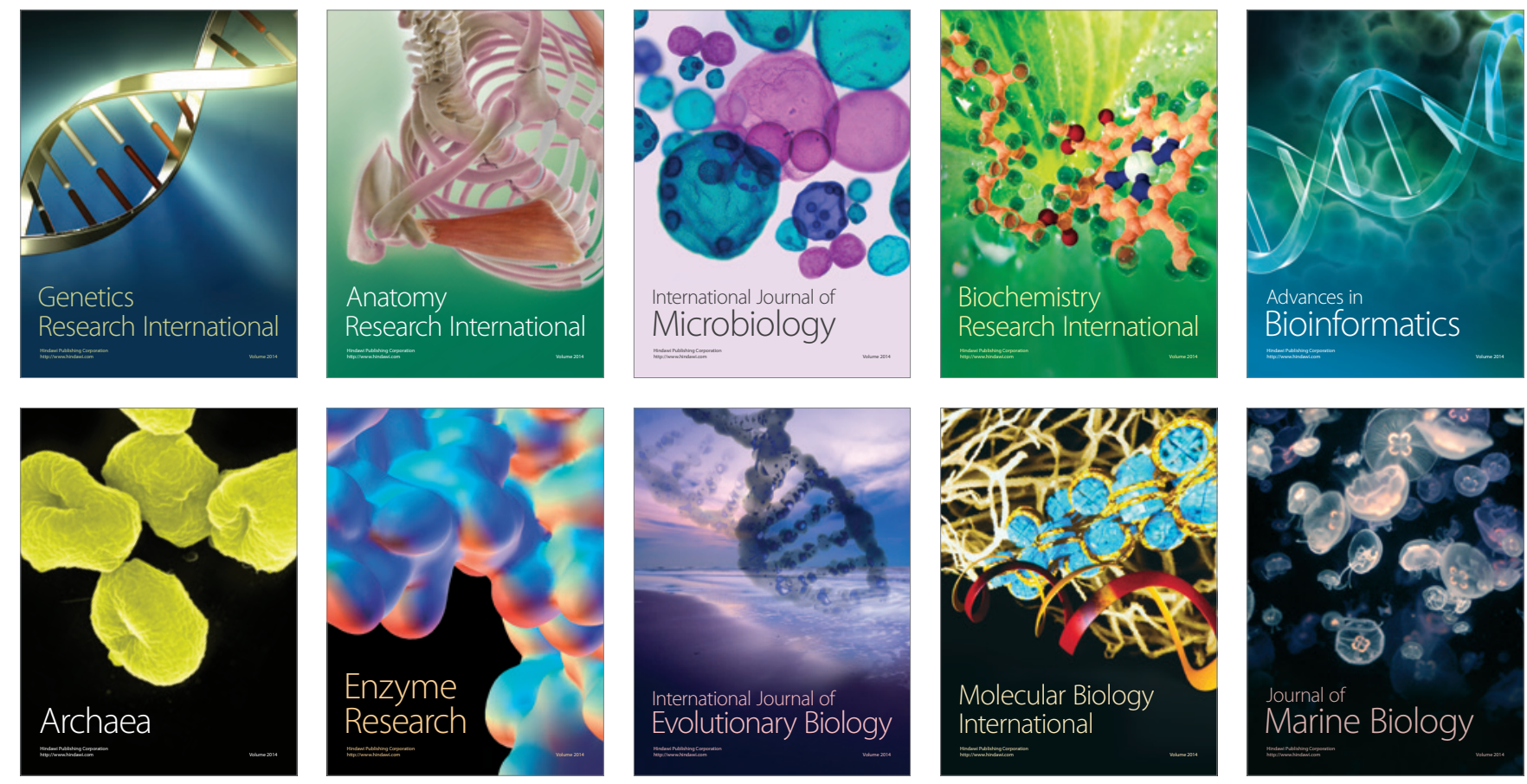